\title{
Interplay of Dirac Nodes and Volkov-Pankratov Surface States in Compressively Strained HgTe
}

\author{
David M. Mahler, ${ }^{1}$ Julian-Benedikt Mayer, ${ }^{2}$ Philipp Leubner, ${ }^{1}$ Lukas Lunczer, ${ }^{1}$ Domenico Di Sante, ${ }^{2}$ Giorgio Sangiovanni, ${ }^{2}$ \\ Ronny Thomale, ${ }^{2}$ Ewelina M. Hankiewicz, ${ }^{2}$ Hartmut Buhmann, ${ }^{1}$ Charles Gould, ${ }^{1}$ and Laurens W. Molenkamp ${ }^{1, *}$ \\ ${ }^{1}$ Physikalisches Institut der Universität Würzburg, 97074 Würzburg, Germany \\ and Institute for Topological Insulators, 97074 Würzburg, Germany \\ ${ }^{2}$ Institut für Theoretische Physik und Astrophysik, Universität Würzburg, 97074 Würzburg, Germany
}

(Received 19 September 2018; revised manuscript received 19 June 2019; published 26 August 2019)

\begin{abstract}
Preceded by the discovery of topological insulators, Dirac and Weyl semimetals have become a pivotal direction of research in contemporary condensed matter physics. While easily accessible from a theoretical viewpoint, these topological semimetals pose a serious challenge in terms of experimental synthesis and analysis to allow for their unambiguous identification. In this work, we report on detailed transport experiments on compressively strained $\mathrm{HgTe}$. Because of the superior sample quality in comparison to other topological semimetallic materials, this enables us to resolve the interplay of topological surface states and semimetallic bulk states to an unprecedented degree of precision and complexity. As our gate design allows us to precisely tune the Fermi level at the Weyl and Dirac points, we identify a magnetotransport regime dominated by Weyl/Dirac bulk state conduction for small carrier densities and by topological surface state conduction for larger carrier densities. As such, similar to topological insulators, $\mathrm{HgTe}$ provides the archetypical reference for the experimental investigation of topological semimetals.
\end{abstract}

DOI: 10.1103/PhysRevX.9.031034

The discovery of topological insulators has inspired a remarkably broad interest in materials whose band structures exhibit relativistic properties. The effects of a linear dispersion in one-dimensional edge channels of quantum spin Hall insulators [1], as well as in two-dimensional surface states of three-dimensional topological insulators $[2,3]$, have already been extensively studied. The implications of a linear band dispersion in three-dimensional conductors, however, have only recently begun to be explored. Such materials, dubbed Dirac or Weyl semimetals, represent a condensed matter realization of the Weyl/ Dirac equations, and may provide an environment for studying the properties of quasiparticles which have been postulated, but not yet unambiguously demonstrated, to exist in nature.

In many of these materials [4], the Weyl or Dirac band crossing is caused by a band inversion, and is intimately connected to the point group symmetry of the crystal lattice. This lends similarities to the prototypical setup of topological insulators. In fact, in both the alkali pnictide

\footnotetext{
*Corresponding author. molenkamp@physik.uni-wuerzburg.de

Published by the American Physical Society under the terms of the Creative Commons Attribution 4.0 International license. Further distribution of this work must maintain attribution to the author(s) and the published article's title, journal citation, and DOI.
}

Subject Areas: Condensed Matter Physics, Topological Insulators

$\left[A B_{3}\right.$, where $\left.A=(\mathrm{Na}, \mathrm{K}, \mathrm{Rb}), \quad B=(\mathrm{As}, \mathrm{Sb}, \mathrm{Bi})\right]$ and $\mathrm{Cd}_{2} \mathrm{As}_{3}$ families that boast a number of important Weyl/ Dirac compounds the inversion occurs between metallic $s$-like and chalcogenic $p$-like orbitals, a situation very similar to that found in $\mathrm{HgTe}$. The correspondence in terms of band structure between these compounds and $\mathrm{HgTe}$ has indeed been known since the 1970s [5]. The common motif is that, for the alkali pnictides and $\mathrm{Cd}_{2} \mathrm{As}_{3}$, the $p$-like $j=$ $3 / 2$ bands ( $\Gamma_{8}$ in the $T_{d}$ point group) cross and yield Dirac (or Weyl) points, while in HgTe the $\Gamma_{8}$ bands just touch, which derives from the higher (zinc blende) point group symmetry of the HgTe crystal. Small crystal distortions from the zinc blende symmetry, as present in Weyl/Dirac semimetals, are sufficient to crucially modify the electronic structure at low energies.

In the 1980s, Volkov and Pankratov [6] studied the interface between two semiconductor materials with mutually inverted bands and reported a resulting band structure as depicted in Fig. 1(a). It includes linear dispersing massless surface states - the states that are now interpreted as the defining property of topological insulators - and topologically trivial massive surface states. While the latter are currently commonly referred to as massive VolkovPankratov states [7], the former are often called topological surface states. Here, we use the historically accurate nomenclature of referring to both types of states identified in Ref. [6] as Volkov-Pankratov states, and differentiating between them by qualifying them as either massless or 

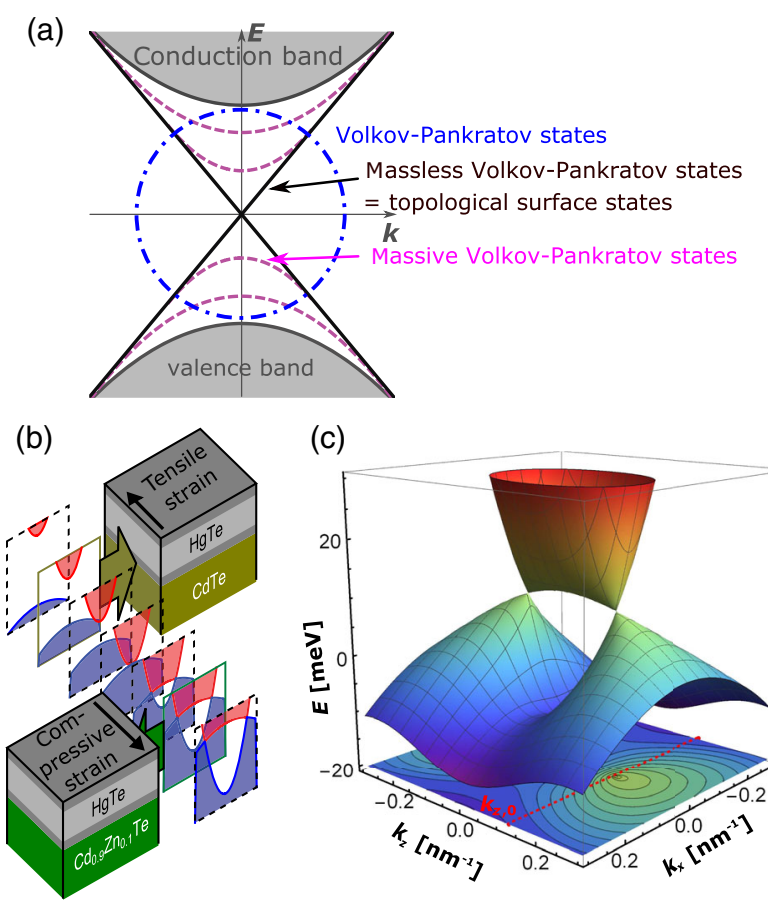

(c)

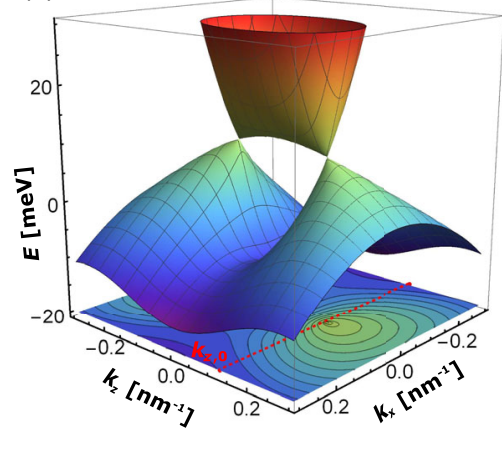

FIG. 1. Panel (a) shows a schematic picture of the band structure at an interface of two semiconductor materials with mutually inverted bulk bands (gray) with two types of interface states (named after the authors of Ref. [6] Volkov-Pankratov states), the massless interface states in black and the massive ones in dashed magenta. (b) Strain-imposed growth of HgTe. Tensile strain is realized by a CdTe substrate, compressive strain by a $\mathrm{Cd}_{0.9} \mathrm{Zn}_{0.1} \mathrm{Te}$ virtual substrate. Plotted as a function of $k_{z}$, i.e., along the growth direction, the band structure profile changes from quadratic band touching for unstrained $\mathrm{HgTe}$ to a topological bulk gap for tensile strain and a pair of linear level crossing for compressive strain. (c) Band structure plot for the compressive strain regime in the $k_{z}-k_{x}$ momentum plane. The bulk inversion asymmetry that would split the Dirac points into Weyl nodes is not accounted for in the calculation.

massive. A generic [8,9] even though experimentally often overlooked or neglected implication of bulk band inversion in Dirac or Weyl materials is the concomitant creation of these same Volkov-Pankratov states at energies even significantly away from the bulk crossing point. As such, while the bulk band structure of these systems exhibits a threedimensional linear dispersion relation, massless VolkovPankratov states continue to support two-dimensional linearly dispersive bands as well. Thus, two- and threedimensional conducting states coexist, and care must be taken in transport experiments to unambiguously assign any feature observed in the conduction profile of the sample to its individual origin.

As an additional challenge for the experimental analysis, Weyl and Dirac materials typically have a high carrier density - as a consequence, they are difficult or sometimes even impossible to gate. Moreover, because proper lithographic methods and thin layer approaches have yet to be developed, primitive contacting (such as needles and conducting glues) and patterning methods are usually employed, giving rise to many potential measurement artifacts resulting from inhomogeneous current distribution, which becomes even further enhanced by the application of magnetic fields. Such effects, sometimes summarized by the expression current jetting [10-12], are, for instance, known to lead to inaccurate mobility measurements. Altogether, such concerns have cast significant doubt on the reliability of many of the early experiments on Dirac and Weyl materials [4].

The synoptic view of the aforementioned observations strongly suggests that materials of higher quality and more mature synthesis procedures are indispensable to truly discover the enigmatic Dirac and Weyl semimetallic state. In this paper, we report that compressively strained $\mathrm{HgTe}$ is an ideal choice for such an undertaking. It can be grown with high crystalline quality by molecular beam epitaxy (MBE), leading to exceptionally low inherent carrier densities. Furthermore, we can use well-established lithographic techniques to precisely define a Hall-bar structure with low resistance alloyed Ohmic contacts as well as electrostatic gate electrodes. These good contacts and exact device geometries ensure a well-defined homogeneous current distribution. Most importantly, the low intrinsic doping, together with the inclusion of a gate, allows us to controllably adjust the level of the Fermi energy via the carrier density within the band structure, tuning the conductance properties between surface state and bulk Dirac/Weyl node dominated transport. This allows us to confidently attribute transport characteristics to either their bulk or surface origin.

The low-energy dispersion of $\mathrm{HgTe}$ is given by two quadratically dispersing $\Gamma_{8}$ bands. For unstrained bulk $\mathrm{HgTe}$, these bands are degenerate at the $\Gamma$ point, as sketched in Fig. 1(b). Under tensile strain, this degeneracy is lifted [13] due to lowering of the point group symmetry, and a topological bulk gap opens $[3,14]$. The remaining surface conduction stems from the massless Volkov-Pankratov states implied by inversion of the $\Gamma_{8}$ bands with the $\Gamma_{6}$ band, the latter of which, for the unstrained case, is located deep below the Fermi level at $\Gamma$.

Under compressive strain, the $\Gamma_{8}$ degeneracy is likewise lifted, now with the two $\Gamma_{8}$ bands shifting in opposite direction as for tensile strain. This leads to the formation of linear crossing points in the band structure. The in-plane compressive and tensile strain dependence of the bands around $\Gamma$ is visualized in Fig. 1(b), where the red domain highlights the conduction and the blue domain the valence regime. Experimentally, the tensely strained 3D topological insulator phase is accomplished by growth on a CdTe substrate [Fig. 1(b)]. While there is no commercially available substrate with a lattice constant slightly below that of $\mathrm{HgTe}$, compressive strain is experimentally still accessible through a superlattice virtual substrate [15] as 
sketched in Fig. 1(b). The linear crossing points are further demonstrated in Fig. 1(c) via a band structure plot along the $k_{z}-k_{x}$ plane in momentum space. It is the result of a sixband $k \cdot p$ calculation of the low-energy band structure for the compressively strained case, where the $z$ axis denotes the layer growth direction and the $x, y$ axes the in-plane coordinates.

More information on the relation between bulk Dirac nodes and the inversion-induced surface states can be obtained from density functional theory calculations on a semi-infinite thick slab with a tellurium terminated interface to vacuum of compressively strained $\mathrm{HgTe}$ at a realistic value of the in-plane strain (see also Supplemental Material [16]). Figure 2(a) shows the calculated slab dispersion for an extended energy range centered around the Fermi level, exhibiting sharp dispersive surface features $20 \mathrm{meV}$ below and right above the Fermi level, which is related to the inversion of the $\Gamma_{6}$ and $\Gamma_{8}$ bands. The breaking of spatial inversion symmetry in the zinc blende structure splits each Dirac node into four Weyl points [17]. For $\mathrm{HgTe}$, Weyl points of opposite chirality project pairwise onto the (001) surfaces connected by inversion. Starting from two Dirac nodes in the unstrained zinc blende structure, this yields four Weyl points per surface, with a Berry flux monopole charge of \pm 2 . Figure 2(b) highlights the lowenergy dispersion for the two surface-projected Weyl points
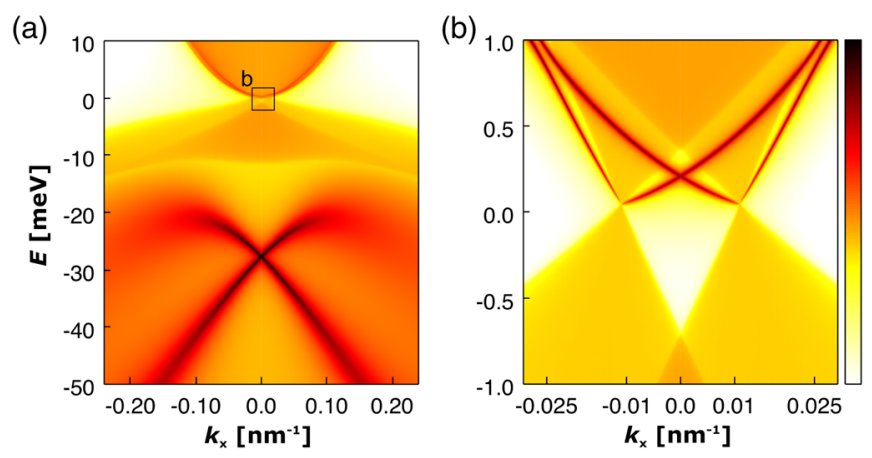

FIG. 2. (a) Band structure along the $k_{x}$ direction of the surface Brillouin zone of a semi-infinite thick slab of compressively strained $\mathrm{HgTe}(\approx 0.3 \%)$ from density functional theory. Spectral features in dark red show prominent surface character, while lighter colours display the continuum of bulk states with relatively weaker projections onto the (001) surface. The sharp dark lines highlight the massless Volkov-Pankratov states and Dirac point stemming from the $\Gamma_{6}-\Gamma_{8}$ band inversion. Because of the sharp interface and the absence of a Hartree potential in this calculations, the massive Pankratov states are shifted to high energies and not visible here. (b) Enlargement of (a) around the Fermi level showing two Weyl points with the same chirality +2 . At large momenta, i.e., far from the Weyl points low-energy physics, the surface states originating from the Weyl nodes are the massless Volkov-Pankratov states that disperse in the unoccupied part of the spectrum [cf. the surface states above the Fermi level in (a)]. The color code identifies the spectral function $A\left(k_{x}, \omega\right)=(-1 / \pi) \operatorname{Im} G_{\text {surf }}\left(k_{x}, \omega\right)$, where $G_{\text {surf }}\left(k_{x}, \omega\right)$ is the momentum and energy resolved surface Green's function. of chirality +2 . Weyl points of opposite chirality are connected by a Fermi arc [18]. The separation in momentum space between the Weyl nodes is estimated to be $\sim 0.02 \mathrm{~nm}^{-1}$. As a consequence, zero-field experiments do not offer sufficient resolution to resolve the small energy and momentum scales at hand. As such, we hereafter consider all bulk transport in this sample to be described by Dirac physics and thus will refer to the energy of the crossing points as the bulk Dirac node. From this perspective, the band structure of compressively strained $\mathrm{HgTe}$ is virtually identical to that of typical 3D Dirac semimetals such as $\mathrm{Cd}_{3} \mathrm{As}_{2}$.

We report experimental results on a 66-nm-thick, compressively strained $\mathrm{HgTe}$ layer, grown on a virtual substrate consisting of a $\mathrm{CdTe} / \mathrm{ZnTe}$ multilayer produced by a combination of MBE and atomic layer epitaxy on a Sidoped GaAs substrate [15]. This CdTe/ZnTe multilayer has a lattice constant between that of CdTe and ZnTe, and can be exactly tuned by setting the $\mathrm{Cd} / \mathrm{Zn}$ ratio. In the present case, a lattice constant of $0.6442 \mathrm{~nm}$ is used to impose a compressive strain of $\approx 0.3 \%$ on the HgTe layer (for details, see the Supplemental Material [16]). To increase sample quality and carrier mobility, two 10-nmthick protective layers of $\mathrm{Cd}_{0.7} \mathrm{Hg}_{0.3} \mathrm{Te}$ are grown below and on top of the HgTe layer.

We pattern the sample using our standard Hall-bar mask and optical lithography process into devices such as the one shown in the inset of Fig. 3. The mask contains two sizes of Hall bars, a larger one with a mesa having a width of $200 \mu \mathrm{m}$ and a separation of the longitudinal voltage leads of $600 \mu \mathrm{m}$, and a smaller one with a width of $10 \mu \mathrm{m}$ and a contact separation of $30 \mu \mathrm{m}$. The HgTe mesas are covered with a 110-nm-thick $\mathrm{SiO}_{2} / \mathrm{Si}_{3} \mathrm{~N}_{4}$ insulator followed by a 100-nm-thick $\mathrm{Au}$ gate electrode on top of a $5-\mathrm{nm} \mathrm{Ti}$ sticking layer. Contacts are fabricated by first using a short dry etching step to provide a clean oxide-free surface for contacting, followed by in situ electron beam evaporation of $50 \mathrm{~nm} \mathrm{AuGe}$ and $50 \mathrm{~nm} \mathrm{Au}$. Two separate devices, each containing Hall bars of both sizes, were investigated in this study, all yielding consistent results with no substantial discrepancy between either the different devices or sample sizes. All measurements are carried out using standard lownoise and low-frequency ac techniques and, unless otherwise noted, are done at $2 \mathrm{~K}$.

We first confirm that we can indeed efficiently adjust the carrier density in our sample as demonstrated by the influence of the gate voltage on the zero-field longitudinal resistance $R_{x x}$ shown in Fig. 3(a). The longitudinal resistance changes by 3 orders of magnitude, from $R_{x x, \max }=$ $19.3 \mathrm{k} \Omega$ around $U_{\text {gate }}=0 \mathrm{~V}$ to $R_{x x}(3 \mathrm{~V})=95 \Omega$. The associated gate-induced change of carrier density in the sample is determined by standard Hall measurements. We find that the density can be tuned from $-10 \times 10^{11} \mathrm{~cm}^{-2}$ ( $p$ type) for $-3 \mathrm{~V}$ up to $10 \times 10^{11} \mathrm{~cm}^{-2}$ ( $n$ type) for $3 \mathrm{~V}$. The maximum of $R_{x x}$ coincides with the lowest total density as well as with the change in the carrier type from 

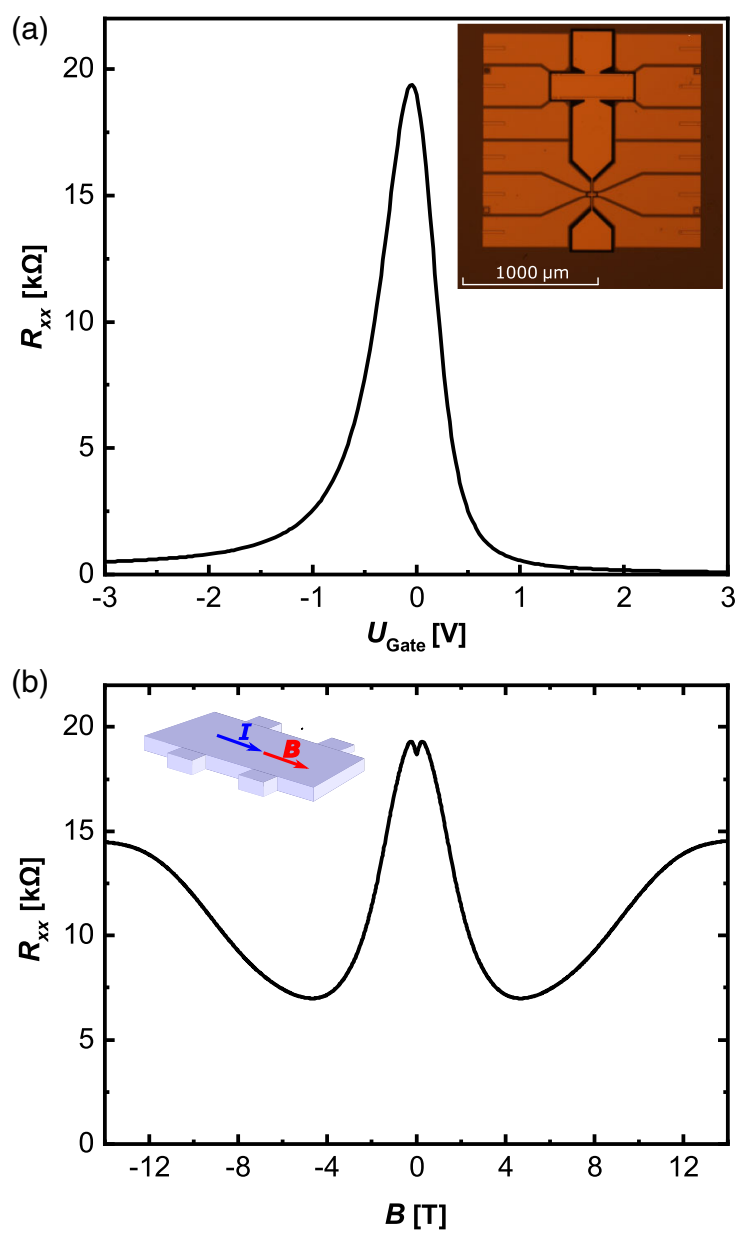

FIG. 3. (a) Longitudinal resistance $R_{x x}$ as a function of the applied gate voltage $U_{\text {gate }}$ for $B=0$. The inset of (a) shows an optical microscope picture of the finished sample. (b) $R_{x x}$ as a function of the applied magnetic field $B$ along the current direction, as indicated by the sketch in the inset, for $U_{\text {gate }}=0 \mathrm{~V}$.

electron to primarily hole transport. From this observation we infer that we can precisely tune the Fermi energy to the bulk Dirac node. Given that our Hall bars consist of three squares, the maximum corresponds to a sheet resistivity value of approximately $6.4 \mathrm{k} \Omega / \square$, and thus of the order of magnitude expected for a diffusive Dirac system [19].

Tuning to minimal carrier density, and consequently moving the Fermi energy to the bulk Dirac node level, the longitudinal resistance $R_{x x}$ versus a magnetic field $B$ applied parallel to the current $I$ is shown in Fig. 3(b). A significant dip is observed as a function of $B$, corresponding to a reduction of up to $\approx 60 \%$ of the value of $R_{x x}$ at $B=0$. Such a negative magnetoresistance contribution is a defining feature implied by the chiral anomaly. Originally conceived as a symmetry violation in quantum field theory in comparison to its classical analogue, the chiral anomaly was first discussed in the context of solid-state systems by Nielsen and Ninomiya [20]. There, the emergence of a chiral charge, i.e., an imbalance between left- and rightmoving Dirac particles, is naturally interpreted to be implied by an external field, as both particle branches are not independent but connected through the crystal band structure. Weyl nodes (and magnetic field-split Dirac nodes) can act as magnetic monopoles in momentum space due to their Berry curvature, with a magnetic charge given by the chirality [21]. A magnetic field parallel to the driving electric field causes a pair of Weyl nodes with different chirality to shift in energy with respect to each other, causing a redistribution of carriers among the nodes. This increases the longitudinal conductivity $\sigma_{x x}$ upon increasing magnetic field strength. A Boltzmann equation calculation yields $\sigma_{x x} \propto B^{2}[22,23]$.

Experimentally, the negative magnetoresistance due to the chiral anomaly contribution is, for certain ranges of magnetic field, often overshadowed by other effects. For small magnetic fields $B$, a minor increase of $R_{x x}$ is observed, which we attribute to weak antilocalization based on its field and temperature dependence $[24,25]$. For large magnetic fields (above $\approx 6 \mathrm{~T}$ ), the chiral anomaly contribution becomes overcompensated to yield a total increase of longitudinal resistance, possibly due to impurityimposed classical mechanisms of magnetotransport [26].

Strong evidence connecting the negative magnetoresistance phenomenon in Fig. 3(b) to the bulk Dirac nodes in the $\mathrm{HgTe}$ band structure derives from the gate voltage dependence of the magnetoresistance data, as presented in Fig. 4. The data of Figs. 3(a) and 3(b) are included as the dashed black line and the $U_{\text {gate }}=0 \mathrm{~V}$ line, respectively. The visualization unambiguously demonstrates that the negative magnetoresistance is strongest, both in absolute numbers and percentagewise, at $U_{\text {gate }} \approx 0 \mathrm{~V}$, corresponding to a Fermi energy close to the bulk Dirac nodes. When the carrier density is increased, the longitudinal resistance $R_{x x}$ at zero field, as well as the magnitude of the negative magnetoresistance, reduces quickly in magnitude. The reduction of the negative magnetoresistance phenomenon with high absolute gate voltage is equivalently observed for both positive $U_{\text {gate }}$ (electron transport) and negative $U_{\text {gate }}$ (hole transport). A slight asymmetry in the negative

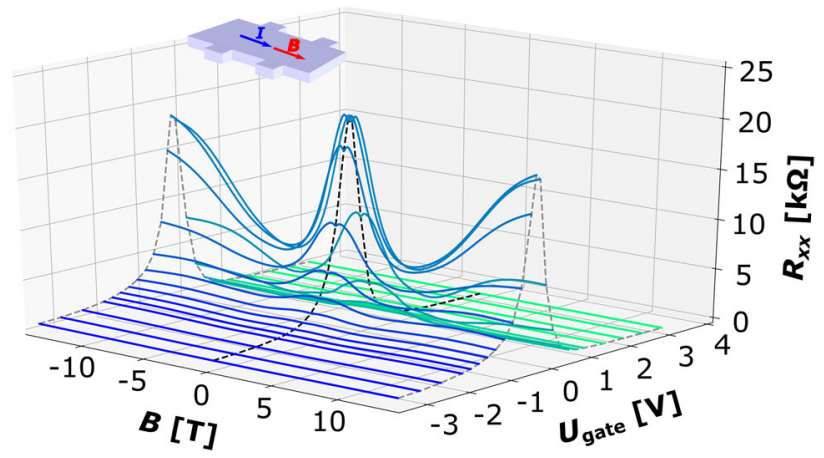

FIG. 4. $\quad R_{x x}$ as a function of the magnetic field applied parallel to the current for different gate voltages. The dashed lines represent $R_{x x}$ as a function of the applied gate voltage for zero (black) and maximum magnetic field ( $\pm 14 \mathrm{~T}$ ) (gray). 
magnetoresistance decay for positive versus negative gate voltages can be attributed to an asymmetry in the electron and hole mobilities.

A further prototypical feature of the chiral anomaly is the implied angular dependence on the magnetic field $B$, as only the magnetic field component $B_{\|}$parallel to the driving electric field $E$ produces an additional current. The angle dependence for $U_{\text {gate }}\left(R_{x x, \max }\right)$ is presented in Fig. 5. In Fig. 5(a) the magnetic field is rotated along the polar angle with $B$ for $\left(\Phi=0^{\circ}\right)$ normal to the plane, and $\left(\Phi=90^{\circ}\right)$ corresponding to $B \| E$. Figure $5(\mathrm{~b})$ shows the $B$ dependence of $R_{x x}$ under variation of the azimuthal angle $\theta$ (in the sample plane). The magnetic field direction is varied from $\theta=0^{\circ}$, representing $B \| E$, to $B$ nearly $\perp I$ for $\theta=85^{\circ}$.

While at higher fields, beyond which the chiral anomaly contribution has saturated, classical positive magnetoresistance contributions take over, both parts of Fig. 5 show that the amplitude of the negative magnetoresistance depends only on the component of $B$ along the current direction, as expected for the chiral anomaly. To further confirm the origin of the negative magnetoresistance, a control experiment, on
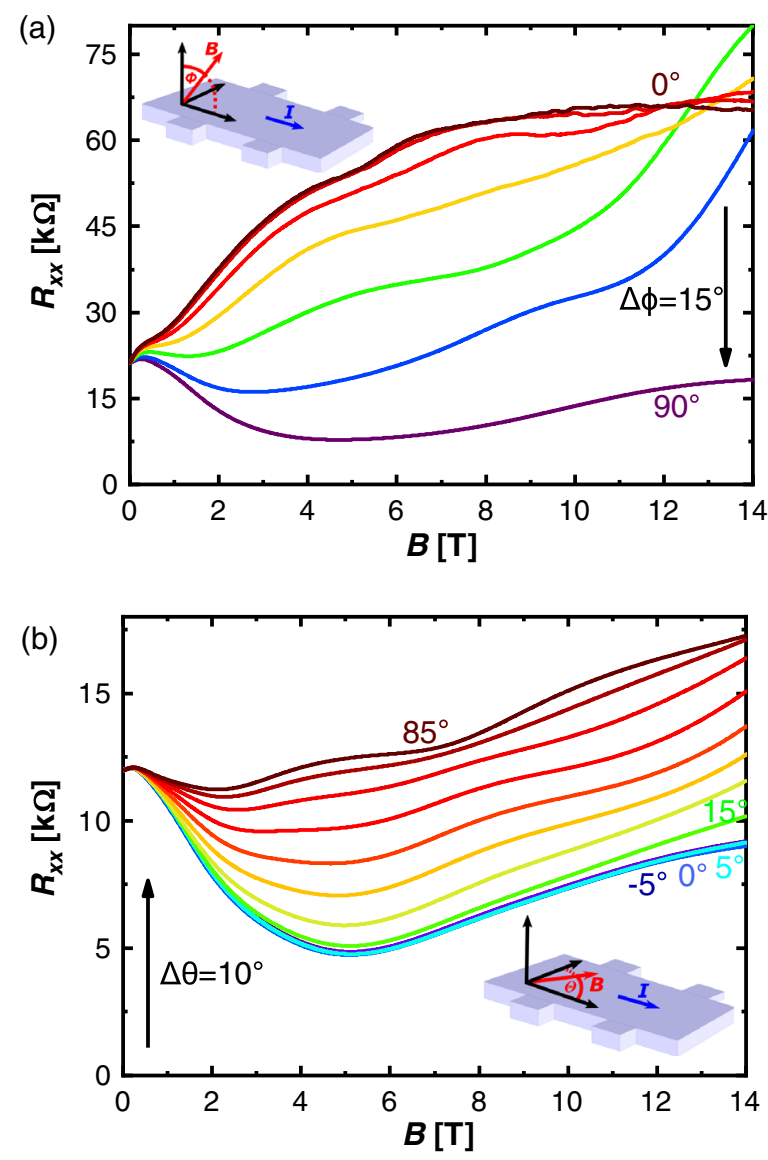

FIG. 5. (a) $R_{x x}$ as a function of the magnetic field $B$ rotated along $\phi$ from perpendicular to the sample plane towards parallel to the current. In (b), $R_{x x}$ as a function of in-plane rotation with angle $\theta$, where $\left(\theta=0^{\circ}\right)$ is parallel to the current and $\theta=85^{\circ}$ nearly perpendicular to the current (inset) at $T=0.3 \mathrm{~K}$. an otherwise identical sample, but with a tensely strained $\mathrm{HgTe}$ layer having a topological insulator band structure, was performed. In that case, only positive magnetoresistance contributions are observed.

We thus conclude that the magnetic field strength and angle as well as gate voltage dependence of the negative magnetoresistance phenomenon discussed so far are fully consistent with the expected behavior driven by a chiral anomaly scenario imposed on left- and right-moving linearly dispersing branches, which comprises conclusive evidence for the existence of Dirac nodes in the bulk band structure of our compressively strained HgTe layer.

As already noted, however, the existence of bulk Dirac nodes in no way precludes the existence of other transport channels. To the contrary, an inverted system with bulk Dirac nodes is generically accompanied by the massless Volkov-Pankratov states. Whether these surfaces contribute to the conductance of the device depends on the location of the Fermi level and the bending of the gate voltage-induced potential over the device [14], and the overall quality of the sample material. The gateability of our sample allows us to explore this coexistence.

Our devices show sharply distinct transport behavior when the gate is used to introduce additional carriers. This is best observed for measurements in a perpendicular magnetic field. For illustration, Fig. 6 depicts transport data at gate voltages of $\pm 4 \mathrm{~V}$ corresponding to highly $n$ type and highly $p$ type. The curves show Shubnikovde Haas oscillations in $R_{x x}$ together with quantum Hall $(\mathrm{QH})$ plateaus. QH plateaus exist only in two-dimensional systems. Consistently, the maxima of the Shubnikov-de Haas oscillations coincide with the transitions between $\mathrm{QH}$ plateaus, suggesting that also the longitudinal resistance $R_{x x}$ is driven by the same two-dimensional transport channel. Since the investigated sample is a threedimensional bulk piece, the two-dimensional character points towards transport carried by a surface state. For this subset of transport contribution, the p-conducting regime [Fig. 6(b)] differs from the $n$-conducting regime [Fig. 6(a)] mainly by overall lower mobility, leading to Landau level (LL) broadening, and consequently less pronounced $\mathrm{QH}$ plateaus. Mobilities of $\mu \approx$ $200000 \mathrm{~cm}^{-2} / \mathrm{Vs}$ are observed for electrons and $\mu \approx$ $30000 \mathrm{~cm}^{-2} / \mathrm{V}$ s for holes. These numbers are comparable to the ones reported for tensely strained $\mathrm{HgTe}$ [27], which is a topological insulator [14]. The accurate quantization of the plateau levels, i.e., exactly equal to the von Klitzing constant to within the experimental accuracy of about $1 \%$, highlights that for these gate voltages, where the surface states are highly populated, the conduction is dominated by surface transport, and bulk Dirac contributions are no longer significant.

An even clearer picture of the interplay between the bulk and surface sources of conduction contributions emerges from the color scale plot of the gate-voltage derivative of 

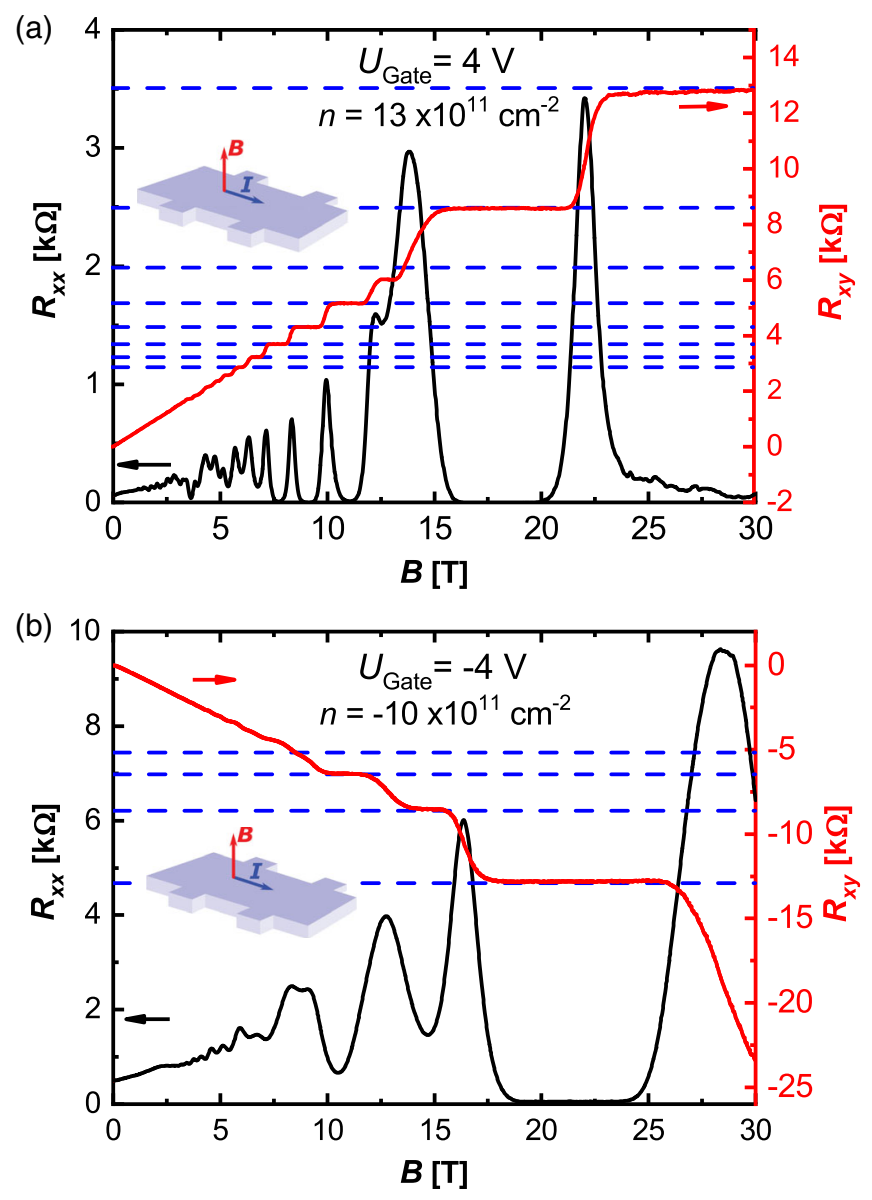

FIG. 6. Longitudinal resistance $R_{x x}$ and Hall resistance $R_{x y}$ as a function of the out of plane magnetic field $B$ for high (a) electron and (b) hole densities at $T=0.3 \mathrm{~K}$.

the Hall conductivity as a function of gate voltage and magnetic field, presented in Fig. 7(b). The derivative values of Hall conductivity (designated in color range from green to red) represent the LL dispersion of a two-dimensional system with respect to the magnetic field $B$ and the gate voltage $U_{\text {gate }}$. For holes $\left(U_{\text {gate }}<0 \mathrm{~V}\right.$ ), a regular pattern of Landau levels is observed. The splitting between two subsequent Landau levels alternates between smaller and bigger gaps. A zero quantum Hall index is observed for low carrier densities $\left(U_{\text {gate }} \gtrsim 0 \mathrm{~V}\right)$ between two nearly nondispersive Landau levels, separating the hole and electron regime. The electron transport regime for higher gate voltages $\left(U_{\text {gate }}>0 \mathrm{~V}\right)$ generally shows a regular pattern of Landau levels. The only exception is the "crossing" of two Landau levels where the quantum Hall index with $\nu=$ 4 would be expected. This effect can be ascribed to the overlap of two types of surface state LL fans: the ubiquitous massless Volkov-Pankratov surface states and the massive Volkov-Pankratov states that arise from the high electric field across the $\mathrm{HgTe} /(\mathrm{Hg}, \mathrm{Cd}) \mathrm{Te}$ interfaces [6] and which were recently identified in HgTe-based topological insulators $[7,28]$. A qualitative description is motivated by $6 \times 6$ $k \cdot p$ calculations ( $\Gamma_{8}$ and $\Gamma_{6}$ bands) with hard-wall boundary conditions in the growth direction, including compressive strain and a bulk inversion asymmetry term from density functional theory calculations, as well as a Hartree potential as in Ref. [14]. Figure 7(a) shows the band structure of a 66-nm-thick sample with an applied Hartree potential corresponding to a gate voltage of $-2 \mathrm{~V}\left(n=-4 \times 10^{11} \mathrm{~cm}^{-2}\right)$. Since the gate voltage is applied from the top surface of the system, such a Hartree potential [shown in the inset of Fig. 7(a)] additionally breaks inversion symmetry. The energies of the massless Volkov-Pankratov states (red) of the top and bottom surface therefore split. Additionally, massive Volkov-Pankratov states (blue) form due to the Hartree potential which confines the bulk states, as also discussed in Ref. [7]. For negative gate voltage, the holelike massive VolkovPankratov state crosses the Fermi energy (orange) and thus has the most significant contribution to transport properties at this gate voltage. For positive gate voltage, the massless Volkov-Pankratov states dominate the transport behavior since the density of the massive Volkov-Pankratov states is negligible. These calculations allow us to assign the black (magenta) Landau levels in Fig. 7(b) to massless (massive) Volkov-Pankratov states. To show the evolution of the band structure under gate voltage, we provide additional calculations for $U_{\text {gate }}=0,+2 \mathrm{~V}$ for the 66-nm-thick sample in the Supplemental Material [16]. $k \cdot p$ calculations and experimental data for a 120 -nm-thick sample are presented along with the analogous analysis in the Supplemental Material [16].

From the above detailed analysis, we conclude that our samples display two distinct transport regimes. First, a narrow gate voltage window around the resistance maximum at $U_{\text {gate }} \approx 0 \mathrm{~V}$, where chiral anomaly-driven negative magnetoresistance is observed (light blue traces in Fig. 4). This effect is only expected from odd-dimensional Dirac cones, in our case the threedimensional bulk Dirac cones. Second, at higher gate voltages (i.e., for finite or high-carrier densities) a twodimensional transport regime is identified by an emerging quantum Hall effect due to the topological surface state of the band inverted material, slightly modified by topologically trivial (massive) Volkov-Pankratov surface states of the material [6]. Our observations provide a simple explanation for the recent findings about a quantum Hall effect in $\mathrm{Cd}_{2} \mathrm{As}_{3}$ layers $[29,30]$ and make it evident that extreme care is needed in claiming any contributions from Fermi arcs in the transport properties of Weyl semimetals in general.

To summarize, compressively strained epitaxial $\mathrm{HgTe}$ layers have proven to constitute an ideal platform for controlled and reliable transport experiments on a Weyl/ Dirac semimetal. Our experiments emphasize the crucial role played by the inversion-induced massless VolkovPankratov surface states in this class of topological 

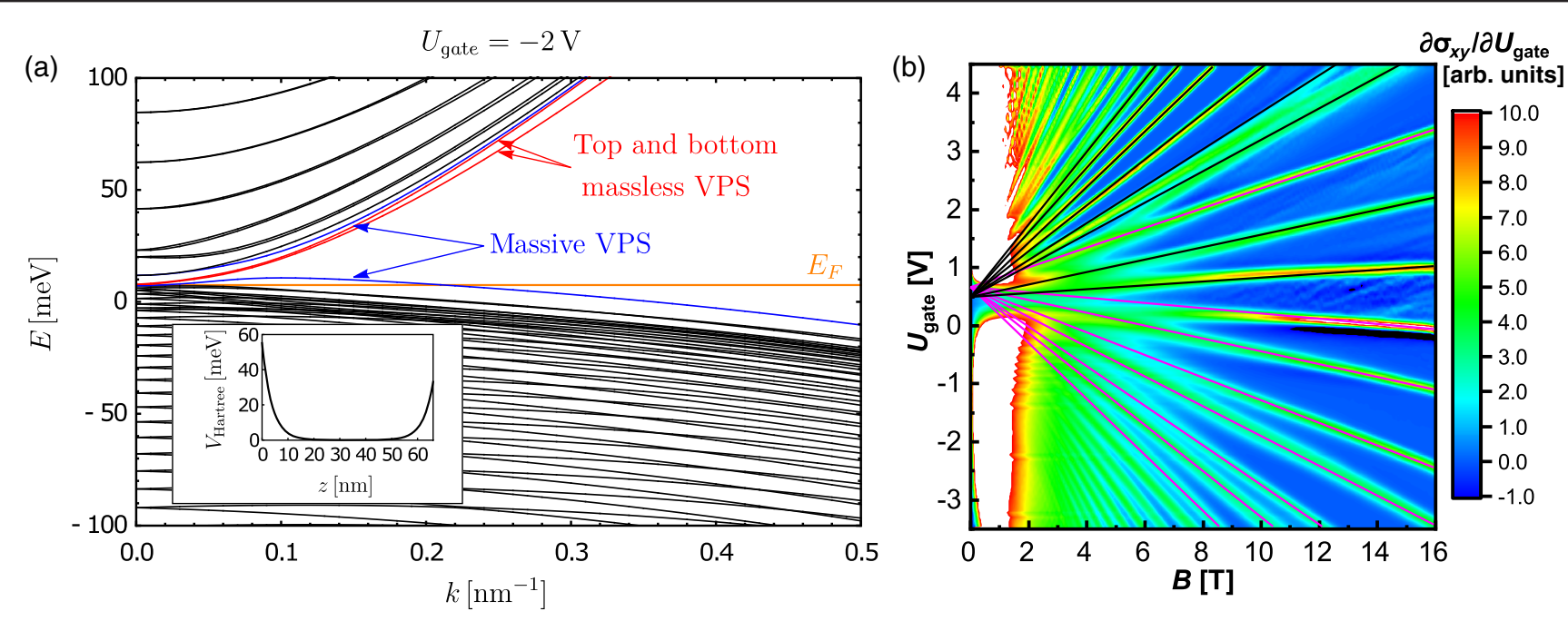

FIG. 7. (a) Calculated $k \cdot p$ band structure for a 66-nm-thick sample including bulk inversion asymmetry terms and a Hartree potential corresponding to an applied gate voltage of $-2 \mathrm{~V}\left(n=-4 \times 10^{11} \mathrm{~cm}^{-2}\right)$. The Hartree potential is presented in the inset of (a). The massless Volkov-Pankratov states are shown in red, while the massive Volkov-Pankratov states are in blue. The bulk subbands are shown in black and the Fermi energy $E_{F}$ is indicated by the orange line. (b) Derivative of the Hall conductivity with respect to the gate voltage $\left(\partial \sigma_{x y} / \partial U_{\text {gate }}\right)$ as a function of the applied gate voltage $U_{\text {gate }}$ and magnetic field $B$ at $T=0.02 \mathrm{~K}$. The solid lines assign the experimental data to two types of Volkov-Pankratov surface (VPS) states: the massless ones (black) and massive ones (magenta).

materials. As supported by our recent results in this direction, compressively strained $\mathrm{HgTe}$ naturally suggest themselves to be an intriguing playground for imposing superconducting proximity effect, where it should be worthwhile to probe the superconducting pairing mechanism at Dirac and Weyl nodes.

The work was supported by the DFG (SFB 1170No. 258499086, Leibniz, and ct.qmat EXC2147 No. 39085490), the EU (ERC-StG-TOCOTRONICSThomale-336012 + ERC-Adv-3TOP-Molenkamp-267436), and the Bavarian ministry of education (ENB and ITI). We acknowledge C. Brüne for useful discussions and F. Schmidt for help with the measurements. The authors gratefully acknowledge the Gauss Centre for Supercomputing e.V. for providing computing time on the GCS Supercomputer SuperMUC at Leibniz Supercomputing Centre (LRZ). Some measurements at high magnetic fields were performed at the HFML, Nijmegen, Netherlands.

[1] M. König, S. Wiedmann, C. Brüne, A. Roth, H. Buhmann, L. W. Molenkamp, X.-L. Qi, and S.-C. Zhang, Quantum Spin Hall Insulator State in HgTe Quantum Wells, Science 318, 766 (2007).

[2] D. Hsieh, D. Qian, L. Wray, Y. Xia, Y. S. Hor, R. J. Cava, and M.Z. Hasan, A Topological Dirac Insulator in a Quantum Spin Hall Phase, Nature (London) 452, 970 (2008).

[3] C. Brüne, C. X. Liu, E. G. Novik, E. M. Hankiewicz, H. Buhmann, Y. L. Chen, X. L. Qi, Z. X. Shen, S. C. Zhang, and L.W. Molenkamp, Quantum Hall Effect from the
Topological Surface States of Strained Bulk HgTe, Phys. Rev. Lett. 106, 126803 (2011).

[4] N. P. Armitage, E. J. Mele, and A. Vishwanath, Weyl and Dirac Semimetals in Three Dimensional Solids, Rev. Mod. Phys. 90, 015001 (2018).

[5] J.-P. Jay-Guerin, M. J. Aubin, and L. G. Caron, The Electron Mobility and the Static Dielectric Constant of $\mathrm{Cd}_{2} \mathrm{As}_{3}$ at 4.2 K, Solid State Commun. 21, 771 (1977).

[6] B. A. Volkov and O. A. Pankratov, Two-Dimensional Massless Electrons in an Inverted Contact, JETP Lett. 42, 4 (1985).

[7] S. Tchoumakov, V. Jouffrey, A. Inhofer, E. Bocquillon, B. Plaçais, D. Carpentier, and M. O. Goerbig, VolkovPankratov States in Topological Heterojunctions, Phys. Rev. B 96, 201302(R) (2017).

[8] B.-J. Yang and N. Nagaosa, Classification of Stable ThreeDimensional Dirac Semimetals with Nontrivial Topology, Nat. Commun. 5, 4898 (2014).

[9] H. Yi et al., Evidence of Topological Surface State in ThreeDimensional Dirac Semimetal $\mathrm{Cd}_{2} \mathrm{As}_{3}$, Sci. Rep. 4, 6106 (2014).

[10] F. Arnold, C. Shekhar, S.-C. Wu, Y. Sun, R. D. d. Reis, N. Kumar, M. Naumann, M. O. Ajeesh, M. Schmidt, A. G. Grushin, J. H. Bardarson, M. Baenitz, D. Sokolov, H. Borrmann, M. Nicklas, C. Felser, E. Hassinger, and B. Yan, Negative Magnetoresistance without Well-Defined Chirality in the Weyl Semimetal TaP, Nat. Commun. 7, 11615 (2016).

[11] K. Yoshida, Transport of Spatially Inhomogeneous Current in a Compensated Metal under Magnetic Fields. III. A Case of Bismuth in Longitudinal and Transverse Magnetic Fields, J. Appl. Phys. 51, 4226 (1980).

[12] R. D. d. Reis, M. O. Ajeesh, N. Kumar, F. Arnold, C. Shekhar, M. Naumann, M. Schmidt, M. Nicklas, and E. Hassinger, On the Search for the Chiral Anomaly in Weyl 
Semimetals: The Negative Longitudinal Magnetoresistance, New J. Phys. 18, 085006 (2016).

[13] L. Liu and W. Leung, Transport Property of Zero-Gap Semiconductors under Tensile Stress, Phys. Rev. B 12, 2336 (1975).

[14] C. Brüne, C. Thienel, M. Stuiber, J. Böttcher, H. Buhmann, E. G. Novik, C.-X. Liu, E. M. Hankiewicz, and L. W. Molenkamp, Dirac-Screening Stabilized Surface-State Transport in a Topological Insulator, Phys. Rev. X 4, 041045 (2014).

[15] P. Leubner, L. Lunczer, C. Brüne, H. Buhmann, and L. W. Molenkamp, Strain Engineering of the Band Gap of HgTe Quantum Wells Using Superlattice Virtual Substrates, Phys. Rev. Lett. 117, 086403 (2016).

[16] See Supplemental Material at http://link.aps.org/ supplemental/10.1103/PhysRevX.9.031034 for further information on the band structure calculations, the strain analysis, as well as measurements on a $120 \mathrm{~nm}$ thick sample.

[17] J. Ruan, S.-K. Jian, H. Yao, H. Zhang, S.-C. Zhang, and D. Xing, Symmetry-Protected Ideal Weyl Semimetal in HgTeClass Materials, Nat. Commun. 7, 11136 (2016).

[18] X. Wan, A. M. Turner, A. Vishwanath, and S. Y. Savrasov, Topological Semimetal and Fermi-Arc Surface States in the Electronic Structure of Pyrochlore Iridates, Phys. Rev. B 83, 205101 (2011).

[19] B. Sbierski, G. Pohl, E. J. Bergholtz, and P. W. Brouwer, Quantum Transport of Disordered Weyl Semimetals at the Nodal Point, Phys. Rev. Lett. 113, 026602 (2014).

[20] H. B. Nielsen and M. Ninomiya, The Adler-Bell-Jackiw Anomaly and Weyl Fermions in a Crystal, Phys. Lett. B 130, 389 (1983).

[21] P. Hosur and X. Qi, Recent Developments in Transport Phenomena in Weyl Semimetals, C.R. Phys. 14, 857 (2013).
[22] D. T. Son and B. Z. Spivak, Chiral Anomaly and Classical Negative Magnetoresistance of Weyl Metals, Phys. Rev. B 88, 104412 (2013).

[23] A. A. Burkov, Negative Longitudinal Magnetoresistance in Dirac and Weyl Metals, Phys. Rev. B 91, 245157 (2015).

[24] H.-Z. Lu and S.-Q. Shen, Weak Antilocalization and Localization in Disordered and Interacting Weyl Semimetals, Phys. Rev. B 92, 035203 (2015).

[25] C.-L. Zhang et al., Signatures of the Adler-Bell-Jackiw Chiral Anomaly in a Weyl Fermion Semimetal, Nat. Commun. 7, 10735 (2016).

[26] R. C. O'Handley, Modern Magnetic Materials: Principles and Applications (John Wiley and Sons, New York, 2000).

[27] A. Jost, M. Bendias, J. Böttcher, E. Hankiewicz, C. Brüne, H. Buhmann, L. W. Molenkamp, J. C. Maan, U. Zeitler, N. Hussey, and S. Wiedmann, Electron-Hole Asymmetry of the Topological Surface States in Strained HgTe, Proc. Natl. Acad. Sci. U.S.A. 114, 3381 (2017).

[28] A. Inhofer, S. Tchoumakov, B. A. Assaf, G. Fève, J. M. Berroir, V. Jouffrey, D. Carpentier, M. O. Goerbig, B. Plaçais, K. Bendias, D. M. Mahler, E. Bocquillon, R. Schlereth, C. Brüne, H. Buhmann, and L. W. Molenkamp, Observation of Volkov-Pankratov States in Topological HgTe Heterojunctions Using High-Frequency Compressibility, Phys. Rev. B 96, 195104 (2017).

[29] C. Zhang, A. Narayan, S. Lu, J. Zhang, H. Zhang, Z. Ni, X. Yuan, Y. Liu, J.-H. Park, E. Zhang, W. Wang, S. Liu, L. Cheng, L. Pi, Z. Sheng, S. Sanvito, and F. Xiu, Evolution of Weyl Orbit and Quantum Hall Effect in Dirac Semimetal $\mathrm{Cd}_{2} \mathrm{As}_{3}$, Nat. Commun. 8, 1272 (2017).

[30] T. Schumann, L. Galletti, D. A. Kealhofer, H. Kim, M. Goyal, and S. Stemmer, Observation of the Quantum Hall Effect in Confined Films of the Three-Dimensional Dirac Semimetal $\mathrm{Cd}_{2} \mathrm{As}_{3}$, Phys. Rev. Lett. 120, 016801 (2018). 